\title{
ON SMALLEST COMPACTIFICATION FOR CONVERGENCE SPACES
}

\author{
C. J. M. RAO
}

\begin{abstract}
In this note we obtain necessary and sufficient conditions for a convergence space to have a smallest Hausdorff compactification and to have a smallest regular compactification.
\end{abstract}

Introduction. A Hausdorff convergence space as defined in [1] always has a Stone-Čech compactification which can be obtained by a slight modification of the result in [3]. But in general this need not be the largest Hausdorff compactification of the space, and in fact it has been pointed out in [4] that the number of distinct maximal Hausdorff compactifications can be quite large. In this note we define the notion of local compactness for a Hausdorff convergence space and show that a Hausdorff noncompact convergence space has a smallest Hausdorff compactification iff the space is locally compact. With a view to obtain a more satisfactory compactification theory for convergence spaces, Richardson and Kent have considered regular compactifications in [4] and have obtained a characterization of the class of convergence spaces for which regular compactifications exist and have shown that each such convergence space has a largest regular compactification. In this note such a convergence space is called an $R$-convergence space, and it has been shown that an $R$-convergence space has a smallest regular compactification iff its pre-topological modification is a locally compact topological space.

1. For terms and results about convergence spaces used in this paper, we refer to [1] and [4]. A convergence space $(S, q)$, where $q$ is the convergence structure will be denoted simply by $S$, and $q$-convergence and $q$-adherence points will be referred to as $S$-convergence and $S$-adherence points respectively. $\dot{x}$ will denote the principal ultrafilter generated by $\{x\}$. For a filter $\mathscr{F}$ on $T$, if its trace on a subset $S$ of $T$ exists, will be denoted by $\mathscr{F} \cap S$, and the filter generated by $\mathscr{F} \cap S$ on $T$ will be denoted by $[\mathscr{F} \cap S]$. $S$ will be called $T$-open if $S$ belongs to every filter on $T$ that $T$-converges

Received by the editors May 10, 1973 and, in revised form, July 6, 1973.

AMS (MOS) subject classifications (1970). Primary 54A05, 54A20; Secondary 54D30, 54D35, 54D45.

Key words and phrases. Convergence space, Hausdorff, regular, locally compact, compact, compactification.

(c. American Mathematical Society 1974 
to some point of $S$. If $T$ and $T^{\prime}$ denote two convergence spaces consisting of the same set and convergence structures $q$ and $q^{\prime}$ respectively, then $T \geqq T^{\prime}$ will mean $q \geqq q^{\prime}$. The same notation $\geqq$ will be used to compare two compactifications of a given space in the usual sense and $=$ will indicate equivalent compactifications. A convergence space $S$ is regular if it is regular in the sense defined in [4], and is therefore also Hausdorff.

1.1. We state a lemma which we will use often without explicitly mentioning it: if $f$ and $g$ are two continuous functions from a convergence space $T_{1}$ to a Hausdorff convergence space $T_{2}$ such that $f$ and $g$ agree on a dense subset $S$ of $T_{1}$, then $f=g$. The proof is simple and therefore omitted.

Let $S$ be a Hausdorff noncompact convergence space and $T=S \cup\{z\}$, where $z$ does not belong to $S$. We make $T$ a convergence space by defining a convergence structure on $T$ as: a filter $\mathscr{F}$ on $T, T$-converges to $x$ in $S$ iff $S$ belongs to $\mathscr{F}$ and $\mathscr{F} \cap S S$-converges to $x$, and for a filter $\mathscr{F}$ on $T, \mathscr{F} T$-converges to $z$ iff $\mathscr{F} \geqq \mathscr{G} \cap \dot{z}$, where $\mathscr{G}$ is any filter on $T$ such that $\mathscr{G} \cap S$ has no $S$-adherence points. It is easy to see that $(T, i)$ is a Hausdorff compactification of $S$, where $i$ is the inclusion map.

Now we give a definition of local compactness for a Hausdorff convergence space which coincides with the usual definition if the convergence space is a topological space, and the compactifications under consideration are Hausdorff topological compactifications.

1.2. Definition. A Hausdorff convergence space is locally compact iff it is open in each of its Hausdorff compactifications.

THEOREM 1.3. A Hausdorff noncompact convergence space has a smallest Hausdorff compactification iff it is locally compact.

Proof. Let $S$ be a Hausdorff noncompact locally compact convergence space and $(T, i)$ be the Hausdorff compactification of $S$ as constructed above; we will show that $(T, i)$ is the smallest Hausdorff compactification of $S$. Let $\left(T^{\prime}, f\right)$ be an arbitrary Hausdorff compactification of $S$, then we define a function $h$ from $T^{\prime}$ onto $T$ as:

$$
\begin{aligned}
h(y) & =i \circ f^{-1}(y), & & \text { for } y \text { in } f(S) \\
& =z, & & \text { for } y \text { in } T^{\prime}-f(S) .
\end{aligned}
$$

We will show that $h$ is continuous. If $\mathscr{F}$ is a filter on $T^{\prime}$ which is $T^{\prime}$ converging to $y$ in $f(S)$, then $S$ being locally compact, $f(S)$ belongs to $\mathscr{F}$ and hence $h(\mathscr{F}) T$-converges to $h(y)$. If $\mathscr{F} T^{\prime}$-converges to $y$ in $T^{\prime}-f(S)$ and: (a) trace of $\mathscr{F}$ on $f(S)$ does not exist, then $T^{\prime}-f(S)$ belongs to $\mathscr{F}$, and hence $h(\mathscr{F})=\dot{z}$ which $T$-converges to $z=h(y)$; (b) trace of $\mathscr{F}$ on $f(S)$ exists, then $\mathscr{F} \cap f(S)$ has no $f(S)$-adherence points and therefore $h(\mathscr{F} \cap f(S))=h(\mathscr{F}) \cap i(S)$ has no $i(S)$-adherence points, and hence 
$h(\mathscr{F}) T$-converges to $z=h(y)$. Since $h \circ f=i$, uniqueness of $h$ follows from the above mentioned lemma and therefore $\left(T^{\prime}, f\right) \geqq(T, i)$.

Next, let $S$ be a Hausdorff noncompact convergence space having a smallest Hausdorff compactification. We first show that if $h$ is a continuous function from a Hausodrff convergence space $T$ onto an arbitrary convergence space such that $h$ restricted to a dense subset $S$ of $T$ is an isomorphism, then $h(T-S) \cap h(S)=\varnothing$. Let $S_{1}=h^{-1} \circ h(S)$; it is sufficient to show that $S_{1}=S$. Now $S \subset S_{1} \subset T, S_{1}$ is Hausdorff, and $S$ is dense in $S_{1}$. Let $g$ be the inverse of the map $h$ restricted to $S$ and $g_{1}$ be $g\left(h \mid S_{1}\right)$, then $g_{1}$ restricted to $S$ is identity, and hence $g_{1}$ is identity from $S_{1}$ to $S_{1}$. Since $g_{1}\left(S_{1}\right) \subset S$, we have $S=S_{1}$. From here we find that if $\left(T_{1}, g_{1}\right)$ and $\left(T_{2}, g_{2}\right)$ are two Hausdorff compactifications of $S$, and $h$ is a continuous function from $T_{1}$ onto $T_{2}$ such that $h \circ g_{1}=g_{2}$, then $h\left(g_{1}(S)\right)=g_{2}(S)$ and $h\left(T_{1}-g_{1}(S)\right)=T_{2}-g_{2}(S)$. Since $(T, i)$ constructed above is a Hausdorff compactification of $S$, the above remarks imply that the smallest Hausdorff compactification of $S$ must be a one point compactification, and therefore $S$ is open in its smallest Hausdorff compactification. From this we see that $S$ is open in each of its Hausdorff compactifications and hence is a locally compact convergence space.

From the proof of the above theorem it is clear that $(T, i)$ is coarser than every Hausdorff compactification of $S$, in which $S$ is open, and therefore, in particular, is coarser than any finite point Hausdorff compactification of $S$. If some Hausdorff compactification of $S$ is coarser than $(T, i)$, then it must be a one point compactification and therefore finer than $(T, i)$, and hence, it can be proved, by using the above mentioned lemma, that this compactification is equivalent to $(T, i)$.

Let $S$ be a locally compact Hausdorff topological space. Let us denote by $\left(T^{\prime}, j\right)$ the one point Hausdorff topological compactification of $S$, and by $(T, i)$ the one point Hausdorff compactification (in the convergence sense) of $S$ as constructed above, then the above remark implies $\left(T^{\prime}, j\right) \geqq(T, i)$. Since $T^{\prime}$ is a compact Hausdorff topological space, Proposition 1.1 in [2] implies $T^{\prime}$ is minimal Hausdorff, and hence Proposition 1.9 in [2] implies $\left(T^{\prime}, j\right)=(T, i)$, because $T$ is a Hausdorff convergence space and $T$ and $T^{\prime}$ are one point compactifications of $S$.

THEOREM 1.4. If $S$ is a locally compact Hausdorff topological space, then $(T, i)$ is its one point Hausdorff topological compactification.

2. As it has been pointed out in [4], not every regular convergence space has a regular compactification. A characterization has been obtained in [4] for regular convergence spaces having a regular compactification. Such a convergence space will be called an $R$-convergence space, and in this section $S$ will always denote an $R$-convergence space. 
If $(P, g)$ is a regular compactification of $S$, then, as shown in [4], $(\pi P, g)$ is a Hausdorff topological compactification of the Tychonoff space $\pi S$.

Let $K$ denote the set of all regular convergence structures $\gamma$ on $P$ which satisfy the following two conditions: $\gamma$ coincides with $\pi P$ relative to ultrafilter convergence, and if $g(S)$ belongs to $\mathscr{F}$, then $\mathscr{F} \gamma$-converges to $y$ in $g(S)$ iff $g^{-1}(\mathscr{F}) S$-converges to $g^{-1}(y)$. These two conditions are consistent because $S$ being an $R$-convergence space, every ultrafilter finer than the neighborhood filter at $x S$-converges to $x$ for all $x$ in $S$ [4]. If $P_{0}$ and $P_{1}$ denote the convergence spaces consisting of the set $P$ equipped with the inf and sup of convergence structures in $K$ respectively, then it is easy to see that $\left(P_{0}, g\right)$ and $\left(P_{1}, g\right)$ are regular compactifications of $S$, $\pi P_{0}=\pi P_{1}=\pi P$ and $P_{1} \geqq P \geqq P_{0}$. Conversely, if $P^{\prime}$ is a regular convergence space such that $P_{1} \geqq P^{\prime} \geqq P_{0}$, then $\pi P_{1}=\pi P_{0}=\pi P^{\prime}$ and $\left(P^{\prime}, g\right)$ is a regular compactification of $S$. For the rest of this section $\left(P_{0}, g\right)$ and $\left(P_{1}, g\right)$ will denote the regular compactifications of $S$ as obtained above for a given regular compactification $(P, g)$ of $S$.

If $(T, f)$ is a Hausdorff topological compactification of the Tychonoff space $\pi S$, then Richardson and Kent have obtained in [4] a regular compactification $\left(T_{1}, f\right)$ of $S$. By construction $T=\pi T_{1}$ and $T_{1}$ is the finest regular convergence space on the set $T$ satisfying the following two conditions: (a) $T_{1}$ coincides with $T$ relative to ultrafilter convergence; and (b) if $f(S)$ belongs to $\mathscr{F}$, then $\mathscr{F} T_{1}$-converges to $y$ in $f(S)$ iff $f^{-1}(\mathscr{F})$ $S$-converges to $f^{-1}(y)$. This leads to the following

PROPOSITION 2.1. If $(P, g)$ is a regular compactification of $S$ and $(T, f) \geqq$ $(\pi P, g)$ as Hausdorff topological compactifications of $\pi S$, then $\left(T_{1}, f\right) \geqq$ $(P, g)$ as regular compactifications of the convergence space $S$.

Proof. Let $h$ be the continuous function from $T$ onto $\pi P$ such that $h \circ f=g$; then we will show that $h$ is continuous from $T_{1}$ onto $P$. If not, there exists a filter $\mathscr{F}_{1}$ on $T$ and $x_{1}$ in $T$ such that $\mathscr{F}_{1} T_{1}$-converges to $x_{1}$, but $h\left(\mathscr{F}_{1}\right)$ does not $P$-converge to $h\left(x_{1}\right)$. We denote by $T_{1}^{\prime}$ a convergence space on the set $T$ having a convergence structure defined as: $T_{1}^{\prime}$ coincides with $T_{1}$ for all $y \neq x_{1}$ in $T$, and $\mathscr{F} T_{1}^{\prime}$-converges to $x_{1}$ iff $\mathscr{F} T_{1}$-converges to $x_{1}$ and $h(\mathscr{F}) P$-converges to $h\left(x_{1}\right) . T_{1}^{\prime}>T_{1}$ implies $T_{1}^{\prime}$ is Hausdorff and $T_{1}^{\prime}$ coincides with $T_{1}$, and therefore with $T$ relative to ultrafilter convergence, and therefore $T_{1}^{\prime}$ is regular. It can be seen that $f(S)$ belongs to $\mathscr{F}$; then $\mathscr{F}$ $T_{1}^{\prime}$-converges to $y$ in $f(S)$ iff $f^{-1}(\mathscr{F}) S$-converges to $f^{-1}(y)$. Hence we get a contradiction, and the result follows.

From this we have the following result, which has been obtained in [4]; if $(T, f)$ is the Stone-Cech topological compactification of the Tychonoff space $\pi S$, then $\left(T_{1}, f\right)$ is the largest regular compactification of $S$. 
If we denote by $T_{0}$ the convergence space consisting of the set $T$ equipped with the inf of all regular convergence structures $\gamma$ on $T$ which satisfy the conditions (a) and (b) above for $\gamma$ in place of $T_{1}$, then $\left(T_{0}, f\right)$ is a regular compactification of $S$ and $\pi T_{1}=\pi T_{0}=T$. This implies $T_{0} \geqq T$. In fact it can be seen that $T_{0}$ is the finest convergence space on the set $T$ having the following properties: if $y$ does not belong to $f(S)$, then $\mathscr{V}_{T}(y)$ $T_{0}$-converges to $y$, where $\mathscr{V}_{T}(y)$ denotes the neighborhood filter in $T$ of the point $y$; if $y$ belongs to $f(S)$ and trace of $\mathscr{V}_{T}(y)$ on $T-f(S)$ exists, then $\mathrm{Cl}_{T} \mathscr{F} \cap\left[\mathscr{V}_{T}(y) \cap(T-f(S))\right] T_{0}$-converges to $y$, where $f(S)$ belongs to $\mathscr{F}$ and $f^{-1}(\mathscr{F}) S$-converges to $f^{-1}(y)$; if $y$ belongs to $f(S)$ and trace of $\mathscr{V}_{T}(y)$ on $T-f(S)$ does not exist, then $\mathrm{Cl}_{T} \mathscr{F} T_{0}$-converges to $y$, where $f(S)$ belongs to $\mathscr{F}$ and $f^{-1}(\mathscr{F}) S$-converges to $f^{-1}(y)$.

Proposition 2.2. If $(P, g)$ is a regular compactification of $S$ and $(\pi P, g) \geqq(T, f)$ as Hausdorff topological compactifications of the Tychonoff space $\pi S$, then $(P, g) \geqq\left(T_{0}, f\right)$ as regular compactifications of the convergence space $S$.

Proof. Let $h$ be the continuous function from $\pi P$ onto $T$ such that $h \circ g=f$. We will show that $h$ is continuous from $P$ onto $T_{0}$. Let $\mathscr{F}$ be a filter on $P$ which $P$-converges to some $y$ not in $f(S)$. Then by construction of $T_{0}, h(\mathscr{F}) T_{0}$-converges to $h(y)$. If $y$ belongs to $g(S)$ and $g(S)$ belongs to $\mathscr{F}$, then clearly $h(\mathscr{F}) T_{0}$-converges to $h(y)$. If $y$ belongs to $g(S)$ and $g(S)$ does not belong to $\mathscr{F}$, then let $\mathscr{G}$ denote the filter $\mathscr{F} \cap(P-g(S))$. Also, if $\mathscr{F}$ has a trace on $g(S)$, we denote it by $\mathscr{H}$. Then $\mathscr{F}=[\mathscr{G}] \cap[\mathscr{H}]$; now by construction of $T_{0}, h(\mathscr{F}) T_{0}$-converges to $h(y)$. Hence $h$ is continuous from $P$ onto $T_{0}$ and therefore the result follows.

For the rest of this section $\left(T_{1}, f\right)$ and $\left(T_{0}, f\right)$ will denote the regular compactifications of $S$ as obtained by the Richardson and Kent method and the above method, respectively, from a given Hausdorff topological compactification $(T, f)$ of the Tychonoff space $\pi S$.

If we write $(P, g) \sim(Q, h)$, where $(P, g)$ and $(Q, h)$ are two regular compactifications of $S$ if $(\pi P, g)=(\pi Q, h)$, that is $(\pi P, g)$ and $(\pi Q, h)$ are two equivalent topological compactifications of the Tychonoff space $\pi S$, then $\sim$ is an equivalence relation on the set of all regular compactifications of $S$.

THEOREM 2.3. If $S$ is an R-convergence space, then each equivalence class of regular compactifications of $S$ has a largest and a smallest member.

Proof. Let $(P, g) \sim(Q, h)$. Since $P_{1} \geqq P \geqq P_{0}$ and $Q_{1} \geqq Q \geqq Q_{0}$, we need only show that $\left(P_{1}, g\right)=\left(Q_{1}, h\right)$ and $\left(P_{0}, g\right)=\left(Q_{0}, h\right)$. The first of these follows from Proposition 2.1 and the second from Proposition 2.2. 
If $\pi S$ is a locally compact topological space and $(T, f)$ is the one point Hausdorff topological compactification of $\pi S$, then from Proposition 2.2 we find that $\left(T_{0}, f\right)$ is the smallest regular compactification of $S$. On the other hand, if $S$ has a smallest regular compactification $(P, g)$, then it is easy to verify that $(\pi P, g)$ is the smallest Hausdorff topological compactification of $\pi S$ and therefore $\pi S$ is a locally compact topological space. This leads to the following.

THEOREM 2.4. If $S$ is an $R$-convergence space, then $S$ has a smallest regular compactification iff $\pi S$ is a locally compact topological space.

The author wishes to thank Professor S. A. Naimpally for his encouragement and also the referee for his valuable suggestions.

\section{REFERENCES}

1. D. C. Kent, Convergence quotient maps, Fund. Math. 65 (1969), 197-205. MR 40 \#3497.

2. D. C. Kent and G. D. Richardson, Minimal convergence spaces, Trans. Amer. Math. Soc. 160 (1971), 487-499. MR 44 \#3279.

3. G. D. Richardson, A Stone-Čech compactification for limit spaces, Proc. Amer. Math. Soc. 25 (1970), 403-404. MR 41 \#992.

4. G. D. Richardson and D. C. Kent, Regular compactifications for convergence spaces, Proc. Amer. Math. Soc. 31 (1972), 571-573. MR 44 \#3290.

Department of Mathematics, Indian Institute of Technology, Kanpur, India 